$\begin{array}{cc}\text { Programa de Pós-Graduação em Engenharia de Produção - PPGEP } & \\ \text { Laboratório de Qualidade de Vida - LaQVida } & \text { REVISTA BRASILEIRA DE } \\ \text { Universidade Tecnológica Federal do Paraná - UTFPR } & \text { Ponta Grossa - PR - Brasil } \\ \text { v. 07, n. 04, out./dez. 2015, p. } 190-209 & \text { QUALIDADE DE VIDA }\end{array}$

\title{
Análise perspectiva da dicotomia entre qualidade de vida no trabalho e produtividade: percepção de gestores
}

\section{Prospective analysis of the dichotomy between quality of work life and productivity: perception of engineers}

\author{
Eliana Aparecida Fagundes Queiroz Bortolozo \\ Universidade Tecnológica Federal do Paraná - UTFPR - Ponta Grossa - Paraná - Brasil \\ bortolozo@utfpr.edu.br \\ Celso Bilynkievycz dos Santos \\ Universidade Tecnológica Federal do Paraná - UTFPR - Ponta Grossa - Paraná - Brasil \\ Universidade Estadual de Ponta Grossa - UEPG - Ponta Grossa - Paraná - Brasil \\ bilynkievycz@,uepg.br \\ Fábio Sprada de Menezes \\ Universidade Tecnológica Federal do Paraná - UTFPR - Ponta Grossa - Paraná - Brasil \\ fabio_1711@yahoo.com.br \\ Luiz Alberto Pilatti \\ Universidade Tecnológica Federal do Paraná - UTFPR - Curitiba - Paraná - Brasil \\ lapilatti@utfpr.edu.br \\ Antonio Augusto de Paula Xavier \\ Universidade Tecnológica Federal do Paraná - UTFPR - Ponta Grossa - Paraná - Brasil \\ augustox@utfpr.edu.br
}

\section{RESUMO}

OBJETIVO: Conhecer a percepção do gestor, especificamente do profissional engenheiro, no tocante aos reflexos dos Programas de Qualidade de Vida no Trabalho (PQVTs).

MÉTODOS: Trata-se de um estudo exploratório. Para a coleta de dados foi construído e validado um instrumento que avalia a percepção de profissionais gestores quanto aos principais benefícios da implantação de um PQVT. Foi adotado o procedimento da amostragem aleatória simples, abrangendo 30 engenheiros, e a pesquisa desenvolveu as etapas do processo de aquisição de conhecimento em base de dados (knowledge discovery in databases - KDD). Para testar o modelo e, a partir da sua taxa de acerto, foram utilizadas técnicas de data mining de cluster.

RESULTADOS: O estudo constatou que apenas 16,66\% dos gestores proporcionam algum PQVT em suas empresas, o que revela um descompasso entre as novas tendências da relação do mundo do trabalho e a efetividade de tais programas. Dos gestores entrevistados, $13,00 \%$ consideraram no máximo sete reflexos positivos dos PQVTs e apontaram, de forma mais frequente no rol de suas próprias prioridades, a diminuição do absenteísmo, o aumento da produtividade e da lucratividade.

CONCLUSÕES: Observa-se que, de modo geral, na implantação de um PQVT, a produtividade é considerada uma variável dependente, sendo a diminuição do absenteísmo e a lucratividade suas 
variáveis intervenientes, a ela associadas. Estes dados demonstram que, do ponto de vista dos gestores, não é possível pensar numa efetiva qualidade de vida no trabalho (QVT) dissociada da produtividade.

PALAVRAS-CHAVE: Qualidade de vida no trabalho. Produtividade. Absenteísmo.

\section{ABSTRACT}

OBJECTIVE: This study aimed to know the vision of the manager, focused on the engineer's, about the reflexes of programs in Quality of Work Life (QWLP) on organizations, from an instrument previously developed and validated.

METHODS: This is an exploratory study. Data have been collected from an instrument that evaluates the perception of professional managers as the main benefits of deploying QWL programs. Simple random sampling has been adopted and 30 engineers were included. In the researching, stages of knowledge acquisition processes have been developed in Database (Knowledge Discovery in Databases - KDD). To test the model, from accuracy rate, Data Mining has been applied.

RESULTS: The results showed that only $16.66 \%$ of managers applied a program in Quality of Work Life in their respective companies, which reveals a mismatch between the new trends regarding the world of labour and the effectiveness of QWLP. Only 13,0 \% of the managers interviewed, have considered seven or less positive impact of the Quality of Life Programs, and included in their list of priorities decreasing absenteeism, increasing productivity and profitability. It can be inferred that by implementing a QWLP, productivity is considered a dependent variable, and decreasing absenteeism and profitability as intervening variables associated with productivity.

CONCLUSIONS: The results demonstrate that managers don't take in consideration that an effective Quality of Work Life program in a disconnected with productivity.

KEYWORDS: Quality of work life. Efficiency. Absenteeism.

\section{Introdução}

No mundo contemporâneo, o fator humano consta como ponto decisivo para o sucesso das organizações; a qualidade de vida no trabalho (QVT) tornou-se uma ferramenta dinâmica, adequada às necessidades das organizações, em várias dimensões, entre elas: a cultura e o clima organizacional; a remuneração e a recompensa; o bem-estar e a segurança no trabalho; o treinamento e o desenvolvimento; a adequação dos recursos; a liberdade e a satisfação no trabalho; o equilíbrio entre a vida profissional e pessoal (KRISHNAKUMAR; SUGAVANESWARI, 2012; SUNDARAM et al., 2012; VARGHESE; JAYAN, 2012).

A QVT tem relação com o nível de felicidade de um indivíduo, no que se refere ao seu trabalho/carreira, considerando suas necessidades, a interpretação da sua carreira e a qualidade do ambiente onde desenvolve suas funções (KRISHNAKUMAR; SUGAVANESWARI, 2012).

Diferentemente de estudos concentrados no desempenho físico e financeiro das organizações, o PQVT denota uma relação complexa entre a satisfação dos funcionários e a eficácia da organização (SUNDARAM et al., 2012). Algumas empresas, dentro do conceito 'gestão de pessoas', associam a satisfação e a valorização do colaborador com a própria qualidade dos seus serviços e produtos (TIMOSSI et al., 2010).

A QVT pode ser discutida a partir de estratégias, operações e aspectos ambientais que promovem e mantêm a satisfação dos funcionários, com vistas a melhorar suas condições de trabalho e sua eficiência (MOHAN; KANTA, 2013). Inclui as estratégias organizacionais que visem à satisfação do trabalhador e sua maior eficácia. Empresas que mantêm PQVT tendem a apresentar 
funcionários mais saudáveis e satisfeitos com suas atividades profissionais (SUNDARAM et al., 2012).

Diversos autores ressaltam que a gestão da QVT vem ganhando destaque nas empresas, contribuindo para que se conte com uma geração de funcionários menos estressados, mais saudáveis, com maior motivação, maior autoestima e melhor relacionamento interpessoal, fatores que levam à queda do absenteísmo e ao aumento da produtividade (GUIDELLI; BRESCIANI, 2010; BACHA et al., 2011; ESFAHANI, SOLTANI, SHIROUYEZAD, 2013; STEPHEN; DHANAPAL, 2012). Quanto mais clara for a identidade do trabalho, maior o nível de qualidade associado com a vida profissional (CHEUNG; TANG, 2009; YEO; LI, 2011; MOHAN; KANTA, 2013).

A aplicação da QVT apresenta dois aspectos relevantes: a muldimensionalidade e a subjetividade. A muldimensionalidade, que permeia a organização sob a forma de melhoria da produtividade, redução de custos e retornos mais elevados, leva em consideração diferentes variáveis; abrange estudos e programas no plano organizacional, técnico e social, a saber: condições, conforto e segurança do trabalho; relações interpessoais; autonomia e liberdade; tomada de decisão, formação e avanço na carreira; suporte dos superiores; gestão de conflitos e, desempenho (YEO; LI, 2011; MOHAN; KANTA, 2013). No plano da subjetividade, entende-se a QVT considerando a percepção da própria pessoa sobre seu estado de saúde e aspectos não-médicos do seu contexto de vida (ATHAYDE; BRITO, 2010).

Segundo Walton $(1973,1974)$, a QVT no plano da subjetividade é um conceito pautado na humanização do trabalho e na responsabilidade social da empresa. Leva em conta o atendimento às necessidades e aspirações do trabalhador. No entanto, apesar dos discursos acadêmicos e das novas políticas de recursos humanos, a discussão que envolve o tema QVT, particularmente no que se refere ao 'aumento de produtividade', denota um desvirtuamento de objetivos, ou seja, da busca do bem-estar dos funcionários no seu ambiente de trabalho e em outras esferas da sua vida, em descompasso, portanto, com o idealizado por Walton. A contradição acontece nas exigências do capital e nas necessidades do trabalhador.

Padilha (2010) admite que poucos trabalhadores conseguem fazer de sua atividade laboral uma fonte financeira aliada à felicidade e à estabilidade, uma vez que novas formas de organização do trabalho são implantadas para aumentar a lucratividade e a competitividade das empresas, e não em benefício dos colaboradores. Para Ferreira, Alves e Tostes (2009), existe desconexão entre os PQVTs e as necessidades dos trabalhadores, fazendo prevalecer um enfoque assistencialista e com baixo grau de coerência entre as diferentes práticas de QVT.

Este cenário gera dúvida sobre qual seria a percepção das empresas e de seus gestores em relação às práticas de QVT. Partindo dos meandros desse questionamento, o presente estudo teve por objetivo conhecer a percepção do gestor, especificamente do profissional engenheiro, no tocante aos reflexos de Programas de Qualidade de Vida no Trabalho (PQVTs). Para a consecução do objetivo foi elaborado, validado e aplicado um instrumento com vistas a identificar a percepção dos entrevistados.

\section{Metodologia}

Trata-se de um estudo exploratório, constituído de duas etapas: elaboração/validação do instrumento, para coleta dos dados, e investigação da percepção do gestor sobre os reflexos dos PQVTs, utilizando-se do instrumento validado.

A amostra de profissionais gestores, composta sob critério aleatório simples, incluiu 30 engenheiros de diferentes áreas, representando 30 diferentes empresas, sendo 20 homens e 10 mulheres, filiados a uma associação de classe da cidade de Ponta Grossa, Paraná, Brasil. Segundo dados do Instituto Paranaense de Desenvolvimento Econômico e Social (2013), a população estimada de Ponta Grossa é de 331.084 habitantes, dos quais 95,7\% residem na zona urbana. O município apresenta um alto Índice de Desenvolvimento Humano (IDH) $(0,763)$, com geração de renda representada, principalmente, pela indústria, seguida por serviços e agricultura. 
Na pesquisa foram desenvolvidas as etapas do processo de aquisição de conhecimento em base de dados (knowledge discovery in databases - KDD) (FAYYAD; PIATETSKY-SHAPIRO; SMYTH, 1996).

O instrumento foi composto de duas partes: a primeira, contendo questões para coleta de informações demográficas; a segunda, composta de perguntas objetivas, com alternativas para respostas dicotômicas e de múltipla escolha (Quadro 1). O processo foi estruturado, mesclando-se diferentes reflexos positivos que os PQVTs possam oferecer, tanto os que priorizam interesses da empresa, como produtividade, lucratividade e absenteísmo, quanto aqueles que demonstram reflexos diretos sobre a pessoa do trabalhador, entre eles bem-estar e saúde.

Quadro 1 - Instrumento de coleta de dados

Parte 1 - Informações Demográficas

1) Ano de formação

2) Sexo

3) Titulação

4) Área de Atuação

Parte 2 - Questões

5a) Conhece algum programa de qualidade de vida no trabalho?

( ) $\mathrm{Sim}$

( ) Não

5b)Em caso afirmativo, qual(is)?

6a) Você ou sua empresa proporciona algum programa de qualidade de vida aos seus colaboradores?

( ) $\mathrm{Sim}$

( )Não

6b) Em caso afirmativo, qual(is)?

7) A partir do pressuposto de que os programas de Qualidade de Vida no Trabalho, conhecidos ou empregados, de forma geral contribuem positivamente em vários aspectos, assinale ENTRE 05 e 10 efeitos que você considera os mais diretos e importantes PARA A EMPRESA, quando da implantação destes programas na organização:

( ) Diminuição de dor e/ou desconforto físico

( ) Melhor disposição para o trabalho e diminuição da fadiga

( ) Melhora na qualidade do sono e repouso

( ) Aumento de sentimentos positivos e otimismo em relação ao futuro

( ) Melhor rendimento profissional, com aumento da produtividade

( ) Capacidade de concentração, aprender novas informações e memorização

( ) Melhora na autoestima e autovalorização

( ) Satisfação com suas capacidades, autoconfiança e capacidade de tomar decisões

( ) Diminuição do absenteísmo

( ) Melhor imagem corporal e aparência

( ) Maior mobilidade e disposição nas atividades da vida cotidiana

( ) Maior lucratividade

( ) Melhora nas relações pessoais e capacidade de dar apoio a outros

( ) Melhora na atividade sexual

( ) Impacto positivo no ambiente doméstico

( ) Melhora nos cuidados de saúde e sociais: disponibilidade e qualidade

( ) Oportunidades de adquirir novas informações e habilidades

( ) Oportunidades de recreação/lazer

( ) Impacto na espiritualidade/religião/crenças pessoais

( ) Maior satisfação com sua saúde

( ) Maior satisfação com sua qualidade de vida

( ) Outro(s):

Fonte: Autoria própria (2014). 


\subsection{Validação do instrumento}

Para avaliar sua qualidade psicométrica de fidedignidade, o método foi aplicado em dois momentos distintos (teste e reteste), por meio do teste estatístico não paramétrico McNemar para variáveis dicotômicas.

O primeiro grupo de dados (teste) foi utilizado como treinamento para o desenvolvimento de modelos; o segundo grupo (reteste), para testar o modelo e, a partir da sua taxa de acerto, avaliar a fidedignidade do instrumento, aplicando-se técnicas de data mining de cluster, seleção de atributos e classificação.

\section{Aquisição de conhecimento potencialmente implícito entre qualidade de vida no trabalho e produtividade}

Nesta etapa da pesquisa, técnicas de data mining de regras de associação foram utilizadas, para identificar conhecimento potencialmente implícito no relacionamento entre as respostas de gestores, quanto aos reflexos dos PQVTs conhecidos ou empregados em suas empresas.

Para utilização das técnicas de data mining, foram percorridas as etapas do processo de KDD (Figura 1), o qual, segundo Michalski e Kaufman (1998), é composto basicamente de três macroetapas: pré-processamento de data mining, data mining e pós-processamento de data mining.

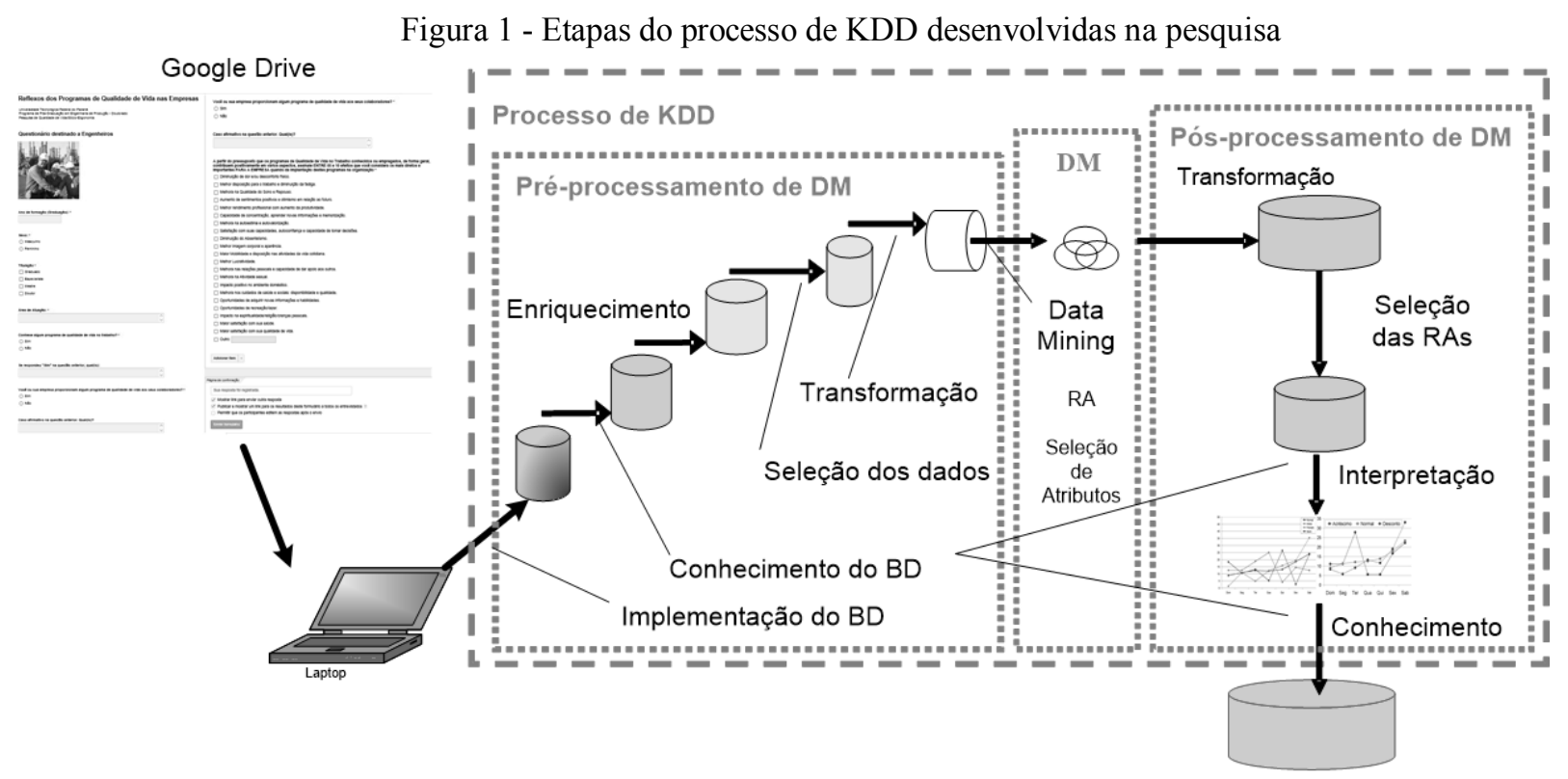

Fonte: Autoria própria (2014).

\subsection{Pré-processamento para data mining}

Esta etapa, realizada em quatro subetapas, teve início com a implementação da base de dados a partir de um instrumento desenvolvido através do software Web Google Drive. Na sequência, passou-se pelo conhecimento da base de dados, pelo enriquecimento da mesma e finalizada com a seleção e a transformação dos dados.

\subsubsection{Conhecimento da base de dados}

O tratamento dos dados, efetuado através dos softwares MS Access e MS Excel, permitiu descrições da amostra, apresentadas na Tabela 1 e na Tabela 2, bem como resultados da frequência relativa dos reflexos dos PQVTs, apontados pelos gestores de equipes de trabalho (Tabela 1, Tabela 2 e Figura 1). O conhecimento da base de dados permitiu identificar a frequência relativa das respostas, para definição do valor mínimo de suporte na configuração do algoritmo minerador.

R. Bras. Qual. Vida, Ponta Grossa, v. 7, n. 4, p. 190-209, out./dez. 2015. 
Tabela 1 - Suporte das variáveis categóricas e estatística de resumo das variáveis numéricas da amostra

\begin{tabular}{|c|c|c|c|}
\hline Variável & Classe & \multicolumn{2}{|c|}{$\%$} \\
\hline \multirow{2}{*}{ Sexo } & Masculino & 66,67 & \multirow{2}{*}{100} \\
\hline & Feminino & 33,33 & \\
\hline \multirow{4}{*}{ Escolaridade } & Graduado & 16,67 & \multirow{4}{*}{100} \\
\hline & Especialista & 40,00 & \\
\hline & Mestre & 10,00 & \\
\hline & Doutor & 33,33 & \\
\hline \multirow{10}{*}{ Área de Atuação } & Construção Civil & 43,33 & \multirow{10}{*}{100} \\
\hline & Ensino & 16,67 & \\
\hline & Tratamento de resíduos & 6,67 & \\
\hline & Projetos e orçamentos & 6,67 & \\
\hline & Engenharia Elétrica & 6,67 & \\
\hline & Engenharia Ambiental & 6,67 & \\
\hline & Saneamento Básico & 3,33 & \\
\hline & Engenharia Química & 3,33 & \\
\hline & Engenharia Mecânica & 3,33 & \\
\hline & Engenharia de Produção & 3,33 & \\
\hline Variável & Estatística de Resumo & \multicolumn{2}{|c|}{ Unidade } \\
\hline Tempo de & Média & \multicolumn{2}{|c|}{19,31 anos } \\
\hline Formação & Desvio Padrão & \multicolumn{2}{|c|}{9,13 anos } \\
\hline
\end{tabular}

Fonte: Autoria própria (2014).

Tabela 2 - Frequência relativa total das opções marcadas

\begin{tabular}{lcc}
\hline \multicolumn{1}{c}{ Questão } & \% Opção Marcada: \\
\cline { 2 - 3 } & Sim & Não \\
\hline Diminuição de dor e/ou de desconforto físico & 46,67 & 53,33 \\
Melhor disposição para o trabalho e diminuição da fadiga & 73,33 & 26,67 \\
Melhora na qualidade do sono e repouso & 40,00 & 60,00 \\
Aumento de sentimentos positivos e otimismo em relação ao futuro & 40,00 & 60,00 \\
Melhor rendimento profissional, com aumento da produtividade & 73,33 & 26,67 \\
Capacidade de concentração, aquisição de novas informações, memorização & 46,67 & 53,33 \\
Melhora na autoestima e autovalorização & 50,00 & 50,00 \\
Satisfação com suas capacidades, autoconfiança e capacidade de tomar decisões & 33,33 & 66,67 \\
Diminuição do absenteísmo & 33,33 & 66,67 \\
Melhor imagem corporal e aparência & 30,00 & 70,00 \\
Maior mobilidade e disposição nas atividades da vida cotidiana & 16,67 & 83,33 \\
Melhor lucratividade & 23,33 & 76,67 \\
Melhora nas relações pessoais e capacidade de dar apoio a outros & 53,33 & 46,67 \\
Melhora na atividade sexual & 6,67 & 93,33 \\
Impacto positivo no ambiente doméstico & 13,33 & 86,67 \\
Melhora nos cuidados de saúde e sociais: disponibilidade e qualidade & 46,67 & 53,33 \\
Oportunidades para aquisição de novas informações e habilidades & 33,33 & 66,67 \\
Oportunidades de recreação/lazer & 26,67 & 73,33 \\
Impacto na espiritualidade/religião/crenças pessoais & 3,33 & 96,67 \\
Maior satisfação com a própria saúde & 26,67 & 73,33 \\
Maior satisfação com sua qualidade de vida & 50,00 & 50,00 \\
Outros: & 0,00 & 100,00 \\
\hline
\end{tabular}

Fonte: Autoria própria (2014). 
Figura 1 - Frequência relativa dos itens apontados como reflexos dos programas de qualidade de vida

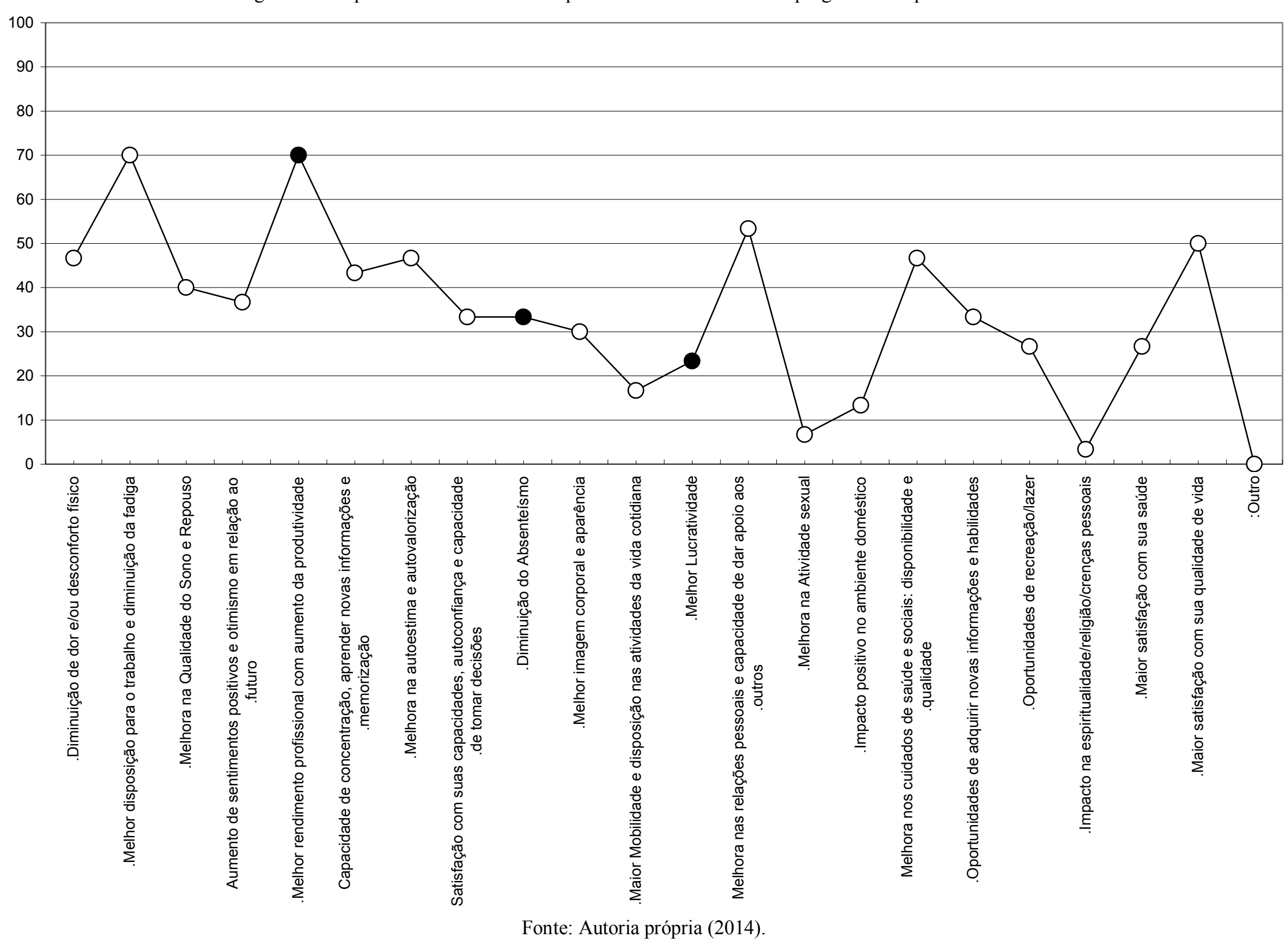

R. Bras. Qual. Vida, Ponta Grossa, v. 7, n. 4, p. 190-209, out./dez. 2015. 


\subsubsection{Enriquecimento da base de dados}

O enriquecimento da base de dados foi realizado com a inclusão de duas variáveis: 'quantidade de opções' e 'classe de resposta'.

Foram definidas duas classes de resposta: 'mínima abrangência' e 'máxima abrangência'. A primeira, atribuída aos registros que apresentaram de cinco a sete opções de respostas; a segunda, conferida aos registros que apresentaram de oito a dez opções.

As Figuras 2 e 3 mostram, respectivamente, a frequência relativa dos reflexos dos PQVTs apontados por estes subgrupos, de abrangência mínima e máxima. 
Figura 2 - Frequência relativa dos itens apontados pela classe menos abrangente, como reflexos dos programas de qualidade de vida no trabalho

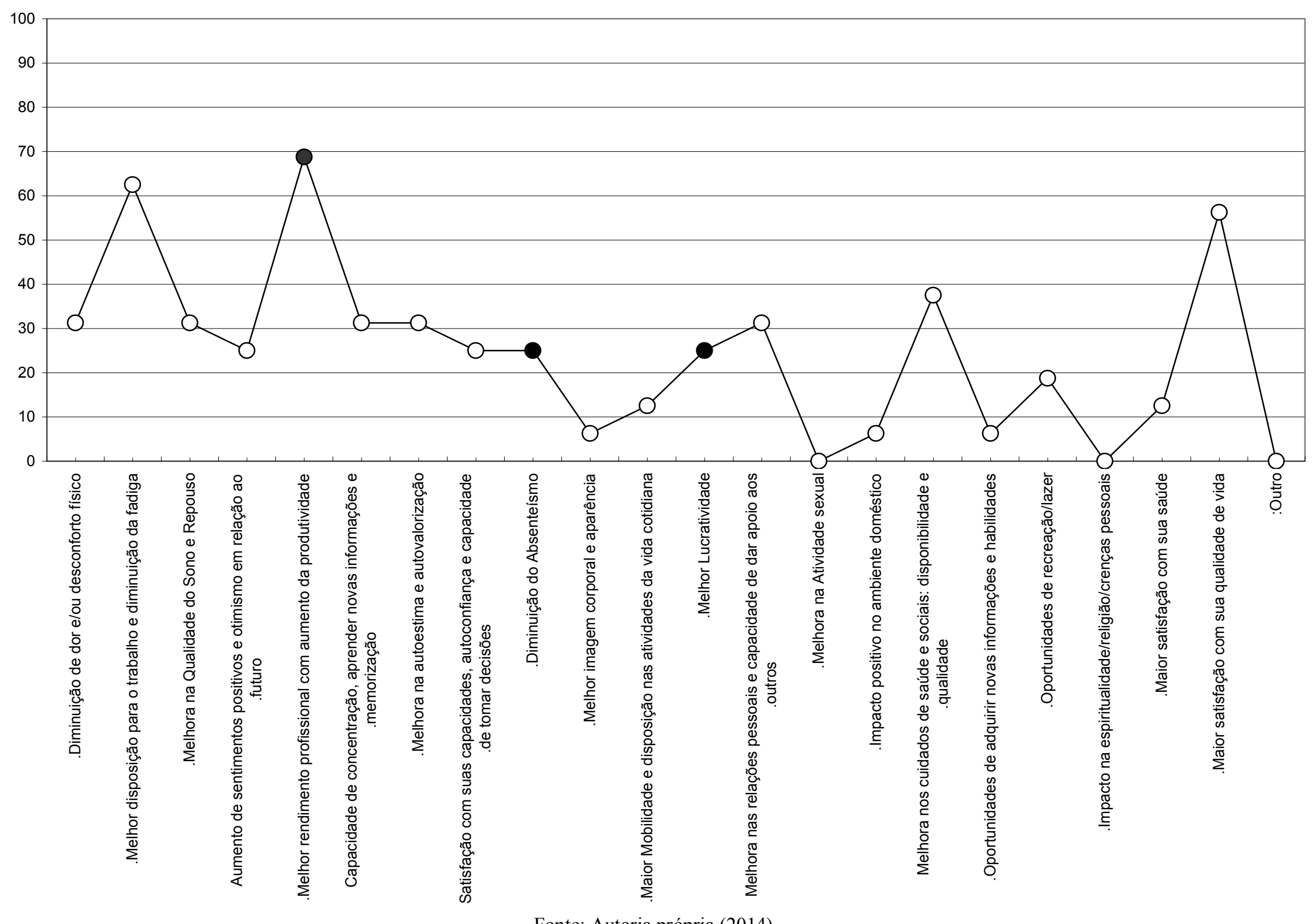

Fonte: Autoria própria (2014)

R. Bras. Qual. Vida, Ponta Grossa, v. 7, n. 4, p. 190-209, out./dez. 2015. 
Figura 3 - Frequência relativa dos itens apontados pela classe mais abrangente, como reflexos dos programas de qualidade de vida no trabalho

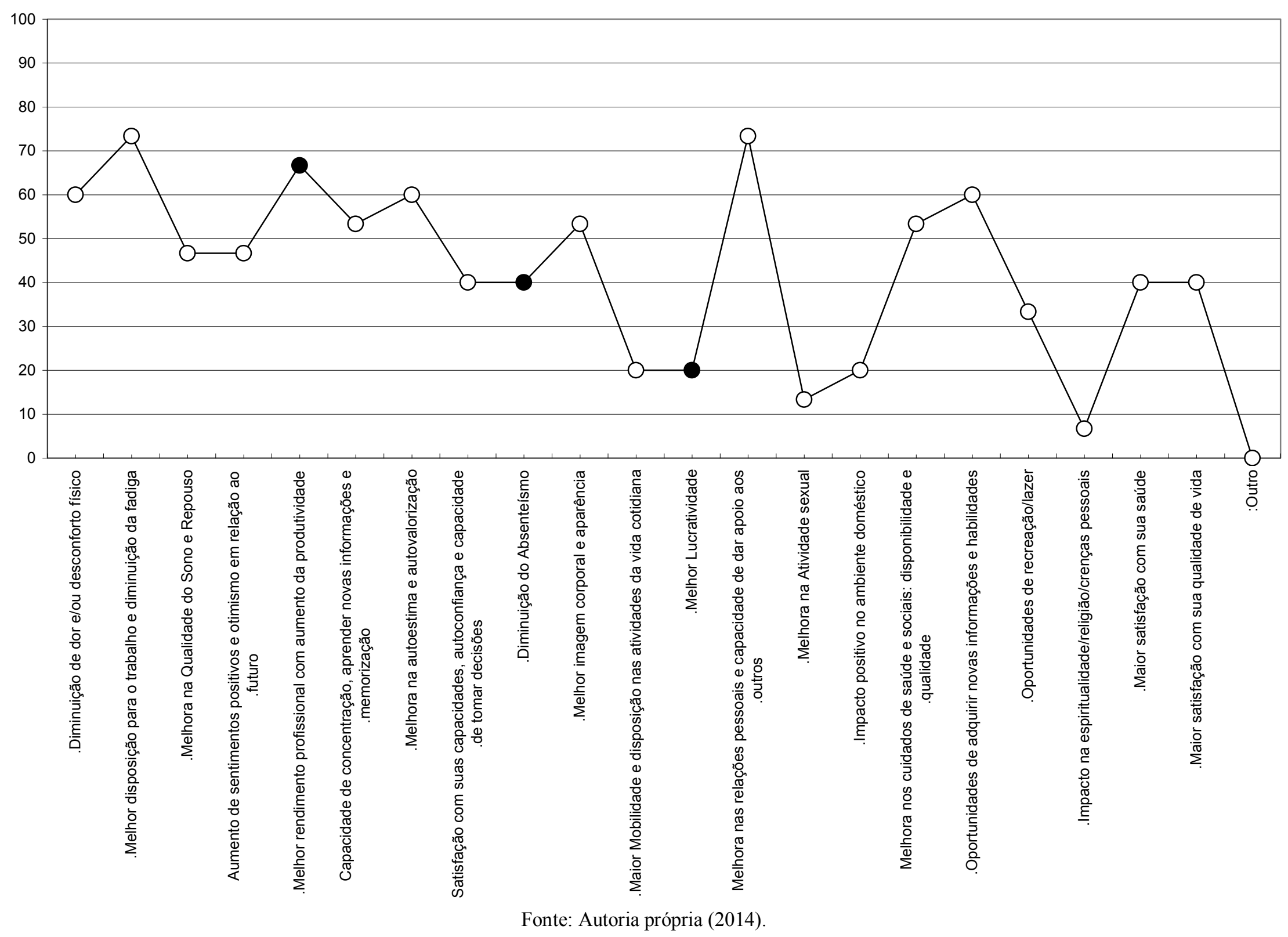

R. Bras. Qual. Vida, Ponta Grossa, v. 7, n. 4, p. 190-209, out./dez. 2015. 


\subsubsection{Seleção e transformação dos dados}

A seleção e transformação dos dados foram realizadas no ambiente WEKA. Inicialmente, configurou-se um driver Open Data Base Connectivity (ODBC) para conectar o software WEKA ao MS Access. Através desta integração foi possível executar Structured Query Language (SQL) no WEKA, efetuando a transformação dos dados para o formato arff, exigido pelo ambiente.

Foram selecionados através de SQL 30 registros com 33 atributos. Não foi necessário realizar a redução de dimensionalidade através de algoritmos de seleção. Alguns atributos foram eliminados por serem muito desbalanceados e não serem considerados atrtibutos metas em possíveis técnicas de classificação.

\subsection{Aplicação da técnica de data mining}

Nesta etapa foram aplicadas técnicas diferentes de data mining, para os mesmos problemas de KDD de descrição e predição.

\subsubsection{Seleção de Atributos}

As técnicas de seleção de atributos buscam identificar os (atributos) que apresentam correlação com uma determinada variável, definida como atributo meta. Geralmente, estas técnicas podem ser utilizadas ainda na etapa de pré-processamento de data mining, para redução de dimensionalidade. Na seleção das variáveis foi utilizado o filtro AttributeSelectio, composto pelo avaliador de atributos CfsSubsetEval (Correlation-based Feature Subset Selecion), através do método de busca Best Fisrt.

Esse algoritmo avalia o valor de um subconjunto de atributos, considerando a capacidade preditiva individual de cada um, juntamente com o grau de redundância entre eles (HALL, 1999). O Quadro 2 resume os atributos previsores (por colunas), juntamente com seus respectivos atributos metas (por linhas). A partir da identificação destas correlações, podem-se desenvolver modelos para a predição de variáveis dependentes em função de variáveis previsoras. 


\begin{tabular}{|c|c|c|c|c|c|c|c|c|c|c|c|c|}
\hline \multirow{2}{*}{\multicolumn{2}{|c|}{ Seleção de Atributos }} & \multicolumn{11}{|c|}{ Atributos Previsores } \\
\hline & & 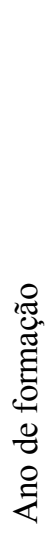 & 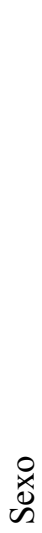 & 苞 & 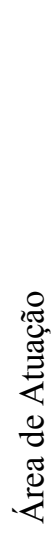 & 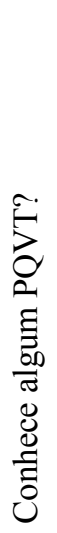 & 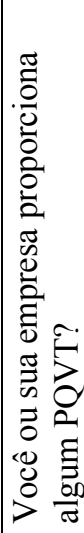 & 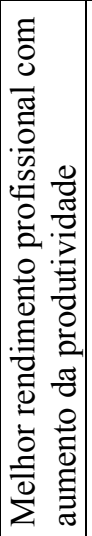 & 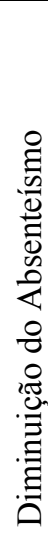 & 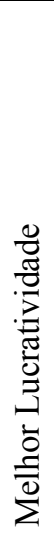 & 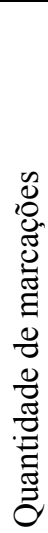 & 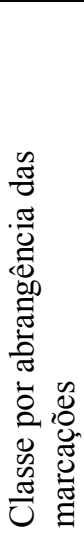 \\
\hline \multirow{11}{*}{ 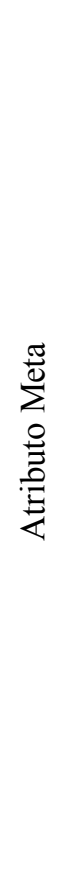 } & Ano de formação & & $\mathrm{x}$ & & & & & & & & & $\mathrm{x}$ \\
\hline & Sexo & & & $\mathrm{x}$ & $\mathrm{x}$ & & & & & & $\mathrm{x}$ & \\
\hline & Titulação & & $\mathrm{x}$ & & $\mathrm{x}$ & & & $\mathrm{x}$ & & & $\mathrm{x}$ & \\
\hline & Área de atuação & $\mathrm{x}$ & & $\mathrm{x}$ & & $\mathrm{x}$ & $\mathrm{x}$ & $\mathrm{x}$ & $\mathrm{x}$ & $\mathrm{x}$ & $\mathrm{x}$ & \\
\hline & Conhece algum PQVT? & & & & $\mathrm{x}$ & & $\mathrm{x}$ & & & & $\mathrm{x}$ & \\
\hline & $\begin{array}{l}\text { Você ou sua empresa } \\
\text { proporciona algum PQVT? }\end{array}$ & & & & $x$ & & $\mathrm{x}$ & & & & $\mathrm{x}$ & \\
\hline & $\begin{array}{l}\text { Melhor rendimento profissional } \\
\text { com aumento da produtividade }\end{array}$ & & & $x$ & $\mathrm{x}$ & & & & & & & \\
\hline & Diminuição do absenteísmo & & & & $\mathrm{x}$ & $\mathrm{x}$ & & & & $\mathrm{x}$ & $\mathrm{x}$ & \\
\hline & Aumento da Lucratividade & & & & $\mathrm{x}$ & & & & $\mathrm{x}$ & & $\mathrm{x}$ & \\
\hline & Quantidade de Marcações & & $\mathrm{x}$ & $\mathrm{x}$ & $\mathrm{X}$ & & $\mathrm{x}$ & & & $\mathrm{x}$ & & $\mathrm{x}$ \\
\hline & $\begin{array}{l}\text { Classe por abrangência das } \\
\text { marcações }\end{array}$ & & & & & & & & & & $\mathrm{x}$ & \\
\hline
\end{tabular}

Fonte: Autoria própria (2014).

\subsubsection{Regras de associação}

Nesta fase foi utilizado o algoritmo Apriori (AGRAWAL; SRIKANT, 1994), o qual permite trabalhos com grande número de atributos, gerando várias alternativas combinatórias entre eles. Realiza buscas contínuas em toda a base de dados, mantendo ótimo desempenho em termos de tempo de processamento.

A configuração do algoritmo no Ambiente WEKA permitiu definir apenas duas medidas objetivas de interesse: o suporte e mais uma (confiança ou lift ou leverage ou convicção). Foram então definidas as seguintes configurações mínimas: suporte $=0,1$ (equivalente a três registros) e convicção $=1,1$, apontando dependência positiva (BRIN et al., 1997), mantendo-se as demais configurações-padrão.

Como medida subjetiva de interesse, foram selecionadas para geração de regras de associação (RA) apenas as variáveis da Tabela 3, ofuscando-se a classe de negação (não), para impossibilitar a geração de RA que apontasse padrões de não ocorrência, assim diminuindo custos operacionais. 
Tabela 3 - Suporte das opções marcadas

\begin{tabular}{lcc} 
Variáveis/classe & Classe & Suporte \\
\hline Você ou sua empresa proporciona algum programa de qualidade? & Sim & 0,16 \\
Conhece algum programa de qualidade de vida no trabalho? & Sim & 0,40 \\
Admite melhor rendimento profissional com aumento da & Sim & 0,73 \\
produtividade? & Sim & 0,33 \\
Diminuição do Absenteísmo? & Sim & 0,23 \\
Maior Lucratividade? & Mínima & 0,53 \\
Classe de abrangência das marcações & Máxima & 0,47 \\
\hline
\end{tabular}

Fonte: Autoria própria (2014).

Estes procedimentos resultaram na geração 38 RA, das quais 17 são apresentadas no Quadro 3. 
Quadro 3 - Regras de associação com suporte mínimo $=0.1$

1. Diminuição do Absenteísmo=Sim Classe $=\min 4==>$ Conhece algum programa de qualidade de vida no trabalho?=Sim 4 conf:(1) lift:(2.5) lev:(0.08) [2] < conv:(2.4)>

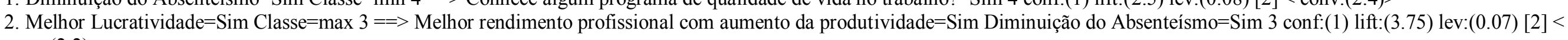
conv:(2.2)>

3. Melhor Lucratividade $=$ Sim Classe $=\max 3==>$ Diminuição do Absenteísmo=Sim 3 conf:(1) lift:(3) lev:(0.07) [2]< conv:(2)>

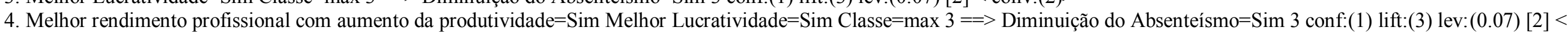
conv:(2)>

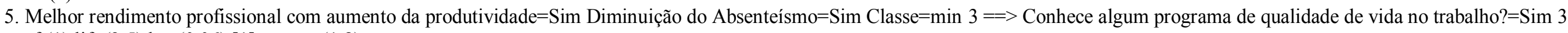
conf:(1) lift:(2.5) lev:(0.06) [1] < conv:(1.8)>

6. Melhor Lucratividade=Sim $7=>$ Diminuição do Absenteísmo=Sim 5 conf:(0.71) lift:(2.14) lev:(0.09) [2] < conv:(1.56)>

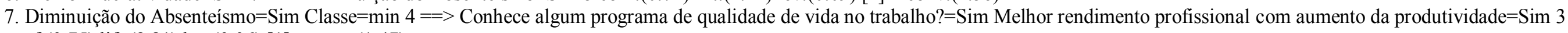
conf:(0.75) lift:(2.81) lev:(0.06) [1] < conv:(1.47)>

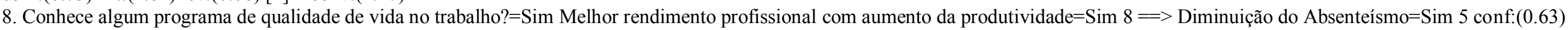
lift:(1.88) lev:(0.08) [2] < conv:(1.33)>

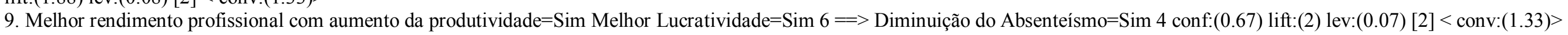

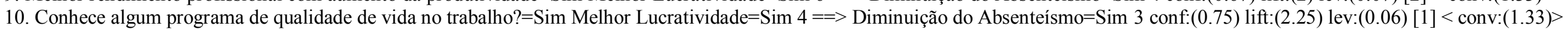

...

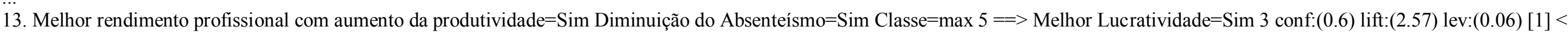
conv:(1.28)>

...

16. Diminuição do Absenteísmo=Sim $10 \Longrightarrow$ Conhece algum programa de qualidade de vida no trabalho?=Sim 6 conf:(0.6) lift:(1.5) lev:(0.07) [2] < conv:(1.2)>

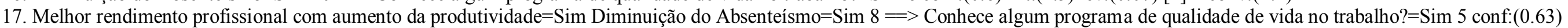
lift:(1.56) lev:(0.06) [1] < conv:(1.2)>

18. Melhor Lucratividade=Sim Classe $=\min 4=\Rightarrow$ Conhece algum programa de qualidade de vida no trabalho?=Sim 3 conf:(0.75) lift:(1.88) lev:(0.05) [1] $<$ conv:(1.2)>

lift:(3.75) lev:(0.07) [2] < conv:(1.2)>

...

31. Conhece algum programa de qualidade de vida no trabalho? $=$ Sim $12==>$ Classe $=\min 8$ conf:(0.67) lift:(1.25) lev:(0.05) $[1]<$ conv:(1.12) $>$

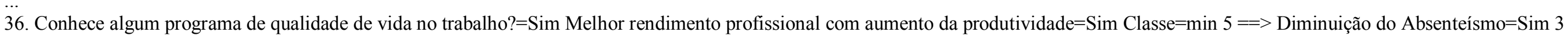
conf:(0.6) lift:(1.8) lev:(0.04) [1] < conv:(1.11)>

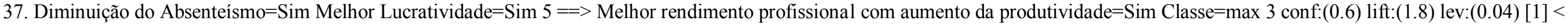
conv:(1.11)>

Fonte: Autoria própria (2014). 


\subsection{Pós-processamento de data mining}

Esta etapa teve início com a seleção das RA, seguida da interpretação dos resultados, e foi finalizada na subetapa de conhecimento adquirido.

\subsubsection{Seleção das regras de associação}

No processo de seleção das RA foram usadas medidas de interesse subjetivas e objetivas. Segundo Silberschatz e Tuzhilin (1995), o grau de interesse de uma RA é avaliado pelo usuário, levando em consideração as suas expectativas e conhecimento prévio dos dados.

Em função da expectativa, foi definida como medida de interesse a subjetividade, a preferência por regras cujo consequente apresentasse variáveis que abrangessem as seguintes dimensões:
a) produtividade;
b) lucratividade;
c) absenteísmo.
As medidas de interesse objetivas adotadas foram:
a) dependência positiva do atributo meta;
b) dependência positiva potencialmente implícita (convicção $<5,0$ );
c) confiança $>=0,60$ (equivalente ao mínimo de 3/5 dos antecedentes);
d) ordenadas pelos maiores valores de: suporte, confiança, convicção, lift e leverage;
e) maior número de itens no antecedente da RA.

\subsubsection{Interpretação}

Como subsídio na interpretação das RA, o ambiente WEKA permite configurar o algoritmo que demonstre a frequência, na base de dados, de todos os elementos envolvidos nas RA (consequentes ou antecedentes), compreendidos pelos valores mínimos de suporte. Nesta etapa, as principais RA foram transformadas em regras de produção, assegurando melhor interpretação, conforme o modelo a seguir (Quadro 4), gerado a partir da primeira RA do Quadro 3, ranqueada pelo algoritmo com a melhor RA.

Quadro 4 - Regra de produção

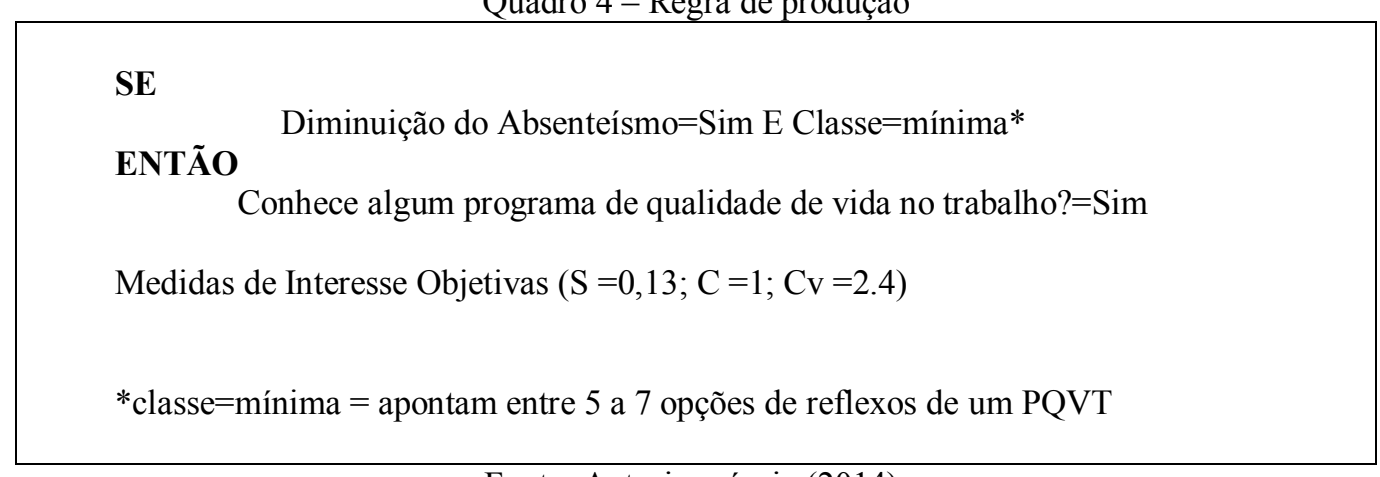

Fonte: Autoria própria (2014).

Esta RA indica que um subgrupo de $13,00 \%$ dos gestores consideraram no máximo sete reflexos positivos dos PQVTs, dentre os quais identificaram a diminuição do absenteísmo e, portanto, conhecem algum PQVT. As medidas de interesse da RA apontam que a possibilidade de 
um gestor conhecer um PQVT é 2,4 vezes maior, caso sinalize, entre um rol de no máximo sete opções, a diminuição do absenteísmo como reflexo positivo do programa para a empresa.

\subsubsection{Aquisição de conhecimento}

Nesta etapa, em que ocorre a transformação do conhecimento potencialmente implícito em explícito, foi realizada a análise dos resultados constantes na Tabela 1 e nas Figuras 3, 4 e 5, além da interpretação das RA de associação (Quadro 1), que são apresentados e discutidos na próxima seção.

\section{Percepção do gestor sobre os reflexos dos PQVT}

A pergunta que norteou a segunda etapa da pesquisa teve por meta identificar qual é a percepção do gestor, no que concerne aos reflexos dos PQVTs. Com a coleta de dados, a partir do instrumento validado com técnicas de data mining de cluster, seleção de atributos e classificação, os resultados indicaram que apenas $16,66 \%$ dos gestores proporcionam um PQVT nas suas empresas, embora $40,00 \%$ deles conheçam algum programa. É uma realidade que demonstra descompasso entre as novas tendências da relação do mundo do trabalho com a efetividade dos PQVTs, igualmente observado por Ferreira, Alves e Tostes (2009), quando estudaram as práticas de QVT em órgãos públicos federais.

Conforme destaca Bacha et al. (2011) e Venson et al. (2013), os PQVTs são relevantes no âmbito de uma organização, tanto sob o aspecto da produtividade individual, quanto da grupal, o que de fato repercute sobre a motivação para o trabalho, adaptação ao ambiente, criatividade e vontade de inovar.

Os padrões de comportamento potencialmente implícitos, por parte dos gestores, em relação às variáveis, foram assim definidos:

a) produtividade representou o apontamento de reflexo mais frequente nas respostas (suporte=73,33\%), em relação com a área de atuação e titulação do gestor;

b) diminuição do absenteísmo foi apontada em 33,33\% dos registros, sendo prioritariamente sinalizada pelo grupo com apontamentos menos abrangentes;

c) lucratividade mostrou frequência relativa de $13,33 \%$, e está associada à área de atuação do gestor, ao número de opções marcadas e ao absenteísmo.

Este estudo apontou, ainda, que para os gestores o aumento da produtividade está associada à diminuição do absenteísmo $(23,33 \%)$ e à lucratividade $(16,66 \%)$.

Segundo Varghese e Jayan (2012), um dos prismas da QVT é o sucesso da organização, enquanto voltada à finalidade de melhorar a eficácia do trabalho. No modelo de Hackman e Oldham (1974), adaptado por Pedroso et al. (2010), no domínio Resultado pessoal e de trabalho, aspectos como 'satisfação com sua produtividade', 'absenteísmo' e 'rotatividade' são apontados e avaliados no contexto da QVT, tendo, porém, como foco o indivíduo, e não a empresa. Para Krishnakumar e Sugavaneswari (2012), trabalhadores que desfrutam de melhor QVT aumentam seu desempenho, o que acaba por refletir positivamente no aumento da produtividade. Celik e Oz (2011) demonstraram uma relação direta entre absenteísmo e trabalhadores com percepção negativa de QVT.

Os resultados apresentados nesta pesquisa demonstram, por parte dos gestores, uma visão enfatizada quanto aos benefícios do PQVT para a empresa, e não no que concerne ao trabalhador, o que resulta em detrimento do propósito primário, que vem a ser o bem-estar do colaborador, sua motivação e satisfação com o trabalho (ROSE et al., 2006). Esta visão sobre QVT, implicando aumento de produtividade, redução de custos e retornos mais elevados, também foi discutida por Ferreira, Alves e Tostes (2009), Mohan e Kanta (2013), Ratnam, Dhanaraj e Rajkumar (2012). De forma similar, Esfahani, Soltani e Shiroyezad (2013) destacaram que PQVTs têm sido implantados 
sob a perspectiva da empresa, como recurso para melhorar a produtividade e meio de valorizar a sua imagem (funcionários e clientes).

Os gestores que fizeram apontamentos menos abrangentes, que destacaram menor número de benefícios dos PQVTs, tendem a priorizar a produtividade ( absenteísmo (13,33\%) e a lucratividade (13,33\%). Por outro lado, aqueles gestores que consideraram maior número de benefícios apontam as mesmas variáveis, mas reduzindo relativamente essas prioridades.

Leite, Ferreira e Mendes (2009) ressaltam que é necessário romper a dicotomia entre bemestar e produtividade, que ainda estão fortemente presentes nos modelos de gestão. Para estes autores, as práticas de QVT devem criar um ambiente social que estimule e propicie a 'produtividade positiva' e as vivências de bem-estar.

A área de atuação está relacionada com maior número de variáveis (Quadro 2), sendo considerada com maior ganho de informação a variável previsora. Logo, torna-se patente que o conhecimento e/ou a aplicação de PQVT está diretamente relacionado à área de atuação, bem como à quantidade de apontamentos de reflexos do próprio PQVT, além do gênero e titulação do gestor. Segundo Krishnakumar e Sugavaneswari (2012), os PQVTs podem variar de organização para organização, dependendo da sua dimensão e da natureza do trabalho.

\section{Considerações finais}

Por meio do instrumento elaborado e validado com a aplicação de técnicas estatísticas de data mining de cluster, seleção de atributos e classificação, foi possível concluir que é baixo o número de PQVTs adotados nas empresas dos gestores pesquisados, embora tenham conhecimento de exemplos desses programas.

Os PQVTs merecem diferentes níveis de importância para os gestores de equipes de trabalho das empresas, de acordo com suas áreas de atuação, sua titulação e seu conhecimento.

O estudo indica uma não dicotomia implícita entre a QVT e a produtividade, ao passo que os gestores de equipes de trabalho apontaram a produtividade como um dos seus principais reflexos. Tal resultado indica que, ao ser implementado um PQVT, a produtividade é considerada uma variável dependente, sendo a diminuição do absenteísmo e a lucratividade suas variáveis intervenientes, a ela associadas.

Assim, o conceito teórico idealizado de QVT, com base na valorização do homem em seu ambiente de trabalho, enquanto ser biopsicossocial, com características individuais e necessidades específicas, para garantir sua qualidade de vida, não vem sendo aplicado em sua essência. Todo o exposto torna evidente o fato de que no mundo do trabalho parece não ser possível pensar numa efetiva QVT dissociada da produtividade.

Vale destacar que - no processo de KDD - foram testadas, para o problema de predição, técnicas de data mining que possibilitassem o desenvolvimento de modelos de classificação. No entanto, estas apresentaram taxas de acerto insatisfatórias, fato este que veio a constituir uma das limitações da presente pesquisa, possivelmente em face do número da amostragem, o que restringe a generalização dos resultados obtidos.

A evidência recolhida sugere a necessidade de nova investigação, aplicando-se o instrumento validado, com grupo maior de gestores de diferentes áreas de formação, que permita corroborar com os resultados obtidos. 


\section{Referências}

AGRAWAL, R.; SRIKANT, R. Fast algorithms for mining association rules. In: VLDB (VERY LARGE DATA BASES) CONFERENCE, 20., 1994, Santiago. Proceedings... Santiago: VLDB, 1994. p. 487-499. Disponível em:

$<$ https://www.it.uu.se/edu/course/homepage/infoutv/ht08/vldb94_rj.pdf>. Acesso em: 19 set. 2015.

ATHAYDE, M.; BRITO, J. Vida, saúde e trabalho: dialogando sobre qualidade de vida no trabalho em um cenário de precarização. Trabalho, Educação e Saúde, v. 7, n. 3, p. 587-597, 2010.

Disponível em: <http://www.scielo.br/pdf/tes/v7n3/09.pdf $>$. Acesso em: 11 ago. 2015. rossef

BACHA, A. M.; LEITE, A. D.; PRADO, R. F. G.; GRASIOTTO, O. R. Políticas públicas na promoção da qualidade de vida e atividade física: a experiência do CAISM. In: GUTIERREZ, G. L.; VILARTA, R.; MENDES, R. T. (Org.). Políticas públicas qualidade de vida e atividade física. Campinas: IPES Editorial, 2011.

BRIN, S.; MOTWANI, R.; ULLMAN, J. D.; TSUR, S. Dynamic itemset counting and implication rules for market basket data. ACM SIGMOD Record, p. 255-264, 1997. rrossef

CELIK, D. A.; OZ, E. U. The effects of emotional dissonance and quality of work life perceptions on absenteeism and turnover intentions among Turkish Call Center Employees. Procedia-Social and Behavioral Sciences, v. 30, p. 2515-2519, 2011. Disponível em: $<$ http://www.sciencedirect.com/science/article/pii/S1877042811023160>. Acesso em: 14 jul. 2015. crossef

CHEUNG, F. Y. L.; TANG, C. S. K. Quality of work life as a mediator between emotional labor and work family interference. Journal of Business and Psychology, v. 24, n. 3, p. 245-255, 2009. Disponível em: <http://link.springer.com/article/10.1007/s10869-009-9103-7\#/page-1>. Acesso em 16 jun. 2015. crossef

ESFAHANI, S. S.; SOLTANI, I.; SHIROUYEZAD, H. Ranking the quality of work life aspects based on competitive advantage factors by quality function deployment approach in Foolad Technique Co. International Journal of Economy, Management and Social Sciences, v. 2, n. 6, p. 244-249, 2013. Disponível em:

$<$ http://waprogramming.com/papers/51ca92edddca41.34562878.pdf $>$. Acesso em: 13 mar. 2015.

FAYYAD, U. M.; PIATETSKY-SHAPIRO, G.; SMYTH, P. From data mining to discovery knowledge in databases. AI Magazine, v. 3, n. 17, p. 37-54, 1996. Disponível em: http://www.aaai.org/ojs/index.php/aimagazine/article/viewArticle/1230. Acesso em: 17 jul. 2015.

FERREIRA, M. C.; ALVES, L.; TOSTES, N. Gestão de qualidade de vida no trabalho (QVT) no serviço público federal: o descompasso entre problemas e práticas gerenciais. Psicologia: teoria e pesquisa, v. 25, n. 3, p. 319-327, 2009. Disponível em:

$<$ http://www.scielo.br/pdf/ptp/v25n3/a05v25n3.pdf>. Acesso em 14 ago. 2015.

GUIDELLI, N. S.; BRESCIANI, L. P. Inovação e qualidade de vida no trabalho: uma visão integrada da gestão a partir de estudo de caso na indústria petroquímica do Grande $\mathrm{ABC}$. Revista de Administração-RAUSP, v. 45, n. 1, p. 57-69, 2010. Disponível em: $<$ www.rausp.usp.br/download.asp?file $=$ v4501057.pdf $>$. Acesso em: 14 mar. 2015.

HALL, M. A. Correlation-based feature selection for machine learning. 1999. $171 \mathrm{f}$. Thesis (Doctorate in Philosophy) - University of Waikato, Hamilton, New Zeland, 1999. 
INSTITUTO PARANAENSE DE DESENVOLVIMENTO ECONÔMICO E SOCIAL. Caderno estatístico Município de Ponta Grossa. 2012. Disponível em: $<$ http:

www.ipardes.gov.br/cadernos/Montapdf.php?Municipio>. Acesso em: 10 dez. 2013.

KRISHNAKUMAR, K. K.; SUGAVANESWARI, P. M. Quality of work life - a phenomenon for organizational success. EXCEL International Journal of Multidisciplinary Management Studies, v. 2, n. 11, p. 220-230, 2012. Disponível em:

$<$ http://zenithresearch.org.in/images/stories/pdf/2012/NOV/EIJMMS/19_EIJMMS_NOV12_KW.pd f>. Acesso 07 ago. 2015.

LEITE, J. V.; FERREIRA, M. C.; MENDES, A. M.. Mudando a gestão da qualidade de vida no trabalho. Revista Psicologia, v. 9, n. 2, p. 109-123, 2009. Disponível em:

$<$ http://pepsic.bvsalud.org/scielo.php?script=sci_arttext\&pid=S1984-66572009000200010>. Acesso: 07 mar. 2015.

MICHALSKI, R.; KAUFMAN, K. Data Mining and Knowledge Discovery: a review of Issues and multistrategy approach. In: MICHALSKI, R. S.; BRATKO, I.; KUBAT, M. (Org.). Machine Learning and Data Mining Methods and Applications. England: John Wiley, 1998.

MOHAN, G. K.; KANTA, K. N. M. Quality of work life: an application of factor analysis. Referred Journal of CMR College of Engineering \& Technology, v. 2, n. 3, p. 4, 2013. Disponível em:

$<$ http://www.indianjournals.com/ijor.aspx?target $=$ ijor:sjm\&volume $=2 \&$ issue $=3 \&$ article $=001>$. Acesso em: 07 mar. 2015.

PADILHA, V. Qualidade de vida no trabalho num contexto de precarização: a panaceia delirante. Trabalho, Educação e Saúde, p. 549-563, 2010. Disponível em:

$<$ http://www.scielo.br/pdf/tes/v7n3/09.pdf>. Acesso em: 11 ago. 2015.

PEDROSO, B.; PILATTI, L. A.; SANTOS, C. B. D.; SANTOS JUNIOR, G. D. Potencial motivador do trabalho: tradução e adaptação cultural do instrumento de Hackman e Oldham.

Revista Produção Online, v. 10, n. 3, p. 670-697, 2010. Disponível em:

$<$ http://producaoonline.org.br/rpo/article/viewFile/533/719>. Acesso em: 14 jul. 2015. Crossef

ROSE, R. C.; BEH, L.; ULI, J.; IDRIS, K. Quality of work life: implications of career dimensions. Journal of Social Sciences, v. 2, n. 2, p. 61-67, 2006. Disponível em:

$<$ http://thescipub.com/PDF/jssp.2006.61.67.pdf $>$. Acesso em: 14 jul. 2015. rossef

RATNAM, S. K.; DHANARAJ, R.; RAJKUMAR, T. T. Quality of work life-how to ensure?

ACADEMICIA: an International Multidisciplinary Research Journal, v. 2, n. 9, p. 214-219, 2012. Disponível em:

$<$ http://www.indianjournals.com/ijor.aspx?target $=$ ijor:aca \&volume $=2 \&$ issue $=9 \&$ article $=017>$. Acesso em: 13 mar. 2015.

SILBERSCHATZ, A.; TUZHILIN, A. On subjective measures of interestingness in knowledge discovery. In: INTERNATIONAL CONFERENCE ON KNOWLEDGE DISCOVERY AND DATA MINING - KDD-95, 1., 1995, Montreal. Proceedings... Palo Alto, California: AAAI, 1995. Disponível em: <https://www.aaai.org/Papers/KDD/1995/KDD95-032.pdf>. Acesso em: 04 maio 2015. 
STEPHEN, A.; DHANAPAL, D. Quality of work life in small scale industrial units: employers and employees perspectives. European Journal of Social Sciences, v. 28, n. 2, p. 262-271, 2012.

Disponível em:

$<$ http://www.theinternationaljournal.org/ojs/index.php?journal=tij\&page=article\&op=view\&path\% 5B $\% 5 \mathrm{D}=447 \&$ path $\% 5 \mathrm{~B} \% 5 \mathrm{D}=\mathrm{pdf}>$. Acesso em: 25 ago. 2015.

SUNDARAM, M. S.; SUBBURAJ, A.; KUMAR, N. M.; GANES, K. S.; SUMATHI, P. Quality of work life among the workers with special reference to manufacturing industries in Sipcot Industrial Park, Sriperumbudur. ZENITH International Journal of Multidisciplinary Research, v. 2, n. 10, p. 201-220, 2012. Disponível em:

$<$ http://www.indianjournals.com/ijor.aspx?target=ijor:zijmr\&volume=2\&issue $=10 \&$ article $=016>$. Acesso em 23 mar. 2015.

TIMOSSI, L. S.; FRANCISCO, A. C.; SANTOS JUNIOR, G.; XAVIER, A. A. P. Análise da qualidade de vida no trabalho de colaboradores com diferentes níveis de instrução através de uma análise de correlações. Produção [online], v. 20, n. 3, p. 471-480, 2010. Disponível em: $<$ http://www.scielo.br/pdf/prod/v20n3/AOP_200901009.pdf >. Acesso em 23 mar. 2015. rossef

VARGHESE, S.; JAYAN, C. Towards understanding the role of quality of work life on job attitudes among law enforcement officers. ZENITH International Journal of Multidisciplinary Research, v. 2, n. 11, p. 1-8, 2012. Disponível em:

$<$ http://www.indianjournals.com/ijor.aspx?target=ijor:zijmr\&volume $=2 \&$ issue $=11 \&$ article $=001>$. Acesso em: 23 jul. 2015.

VENSON, A. B. S.; FIATES, G. G. S.; DUTRA, A.; CARNEIRO, M. L.; MARTINS, C. O recurso mais importante para as organizações são mesmo as pessoas? Uma análise da produção científica sobre qualidade de vida no trabalho (QVT). Revista de Administração da UFSM, v. 6, n. 1, p. 139-156, 2013. Disponível em: < http://cascavel.cpd.ufsm.br/revistas/ojs2.2.2/index.php/reaufsm/article/view/5705>. Acesso em: 16 mar. 2015. Crossef

WALTON, R. E. Quality of working life: what is it? Slow Management Review, v. 15, n. 1, p. 1121, 1973.

WALTON, R. E. Improving the quality of work life. Harvard Business Review. v. 52, n. 3, p. 12, 1974.

YEO, R. K.; LI, J. Working out the quality of work life: A career development perspective with insights for human resource management. Human Resource Management International Digest, v. 19, n. 3, p. 39-45, 2011. Disponível em:

$<$ http://www.emeraldinsight.com/doi/abs/10.1108/09670731111125952>. Acesso em: 14 maio 2015. Crossef

\section{Conflitos de interesse}

Não há nenhum potencial conflito de interesse entre os autores desse trabalho. 